\title{
On the Uniqueness of the Invariant Equilibrium State and Surface Tension
}

\author{
C. Gruber and A. Hintermann* \\ Laboratoire de Physique Théorique, Ecole Polytechnique Fédérale de Lausanne, \\ $\mathrm{CH}$-Lausanne, Switzerland
}

\author{
A. Messager and S. Miracle-Sole \\ Centre de Physique Théorique, C.N.R.S., F-Marseille, France
}

\begin{abstract}
Symmetric Equilibrium States and their properties under duality transformation are investigated. Necessary and sufficient conditions are derived for equilibrium states to be transformed into equilibrium states by duality. It is shown that ferromagnetic systems satisfying those conditions have correlation functions bounded by those corresponding to the $(+)$ and free boundary conditions. It is then proved than any Invariant Equilibrium State of a ferromagnetic system is transformed into an equilibrium state by duality and is thus unique if the states defined by the $(+)$, and free boundary conditions coincide on the symmetric algebra. The existence of surface tension between two pure phases is established.
\end{abstract}

\section{Introduction}

In this paper, we investigate some consequences of the duality transformation ${ }^{1}$ which are of interest for the study of ferromagnetic systems. It was recently established that, for all temperature, there exists a unique, symmetric, translation invariant, equilibrium state for the two-dimensional Ising model [2]; as was suggested in [3] the duality transformation was indeed a key to the proof of this result. The motivation of the following work relies upon the conjecture that for any lattice system, there should be a unique equilibrium state, which is invariant under the full symmetry group of the Hamiltonian, also called "Invariant Equilibrium State". We shall then derive general properties of symmetric equilibrium states and discuss certain consequences of the duality transformation.

In Section 3, we define symmetric equilibrium states by means of the solutions of equations which are well adapted for the study of duality. Necessary and sufficient conditions are then derived in Section 4 for a state to be transformed into an

* Present address : Swiss Institute for Nuclear Research, CH-5234 Villigen, Switzerland

1 Introduced by H. A. Kramers and G. H. Wannier for the Ising model duality is a symmetry property inherent to lattice systems. See for instance [1] and references cited below 
equilibrium state by duality. In particular, for ferromagnetic systems, it is shown that any state satisfying those conditions has correlation functions bounded above and below by those of the states $\omega_{+}$and $\omega_{f}$ defined respectively by the $(+)$ and (free) boundary conditions; it is also proved that any Invariant Equilibrium State yields by duality an equilibrium state of the dual system. It thus follows that the correlation functions of any Invariant Equilibrium State are always bounded by those of $\omega_{+}$and $\omega_{f}$ and therefore the unicity of the Invariant Equilibrium State follows in the case $\omega_{+}=\omega_{f}$. The duality transformation is also applied in Section 5 to the problem of surface tension where we prove that the surface tension between two pure phases is well defined, non positive and bounded below.

To conclude this introduction, we shall note that we have restricted ourselves to the case of HT-LT duality transformations; it has been recently established [4] that for HT-HT as well as LT-LT duality transformation there exists a bijection between symmetric equilibrium states of the system and its dual.

We finally remark that J. L. Lebowitz [18], has recently derived new inequalities which are relevant for the problem of unity of the symmetric invariant equilibrium state. In particular it can be shown that the symmetric invariant equilibrium state is unique whenever the energy density is continuous.

\section{Notation}

We consider a general spin $\frac{1}{2}$ lattice system $\{\mathscr{L}, \mathscr{B}, K\}$ defined by a lattice $\mathscr{L}$, a family $\mathscr{B} \subset \mathscr{P}_{f}(\mathscr{L})$ of bonds, and a real or complex function $K: \mathscr{B} \rightarrow \mathbb{C}$ such that $K(B) \neq\left\{0, \pm i \frac{\pi}{2}, \infty\right\}$ where $K(B)=\frac{1}{k T} J(B)$ and $J(B)$ describe the interaction between the spin at sites $B$.

With any set $\mathscr{C},|\mathscr{C}|$ denotes the cardinality of $\mathscr{C}, \mathscr{P}(\mathscr{C})\left[\right.$ resp. $\left.\mathscr{P}_{f}(\mathscr{C})\right]$ denotes the group defined by the subsets of $\mathscr{C}$ [resp. finite subsets of $\mathscr{C}$ ] together with the product defined by the symmetric difference of sets $X \cdot Y=(X \cup Y) \backslash(X \cap Y)$, $X, Y \in \mathscr{P}(\mathscr{C})$. For any $X \in \mathscr{P}(\mathscr{C})\left[\right.$ resp. $\left.\mathscr{P}_{f}(\mathscr{C})\right] \sigma_{X}$ denotes the function on $\mathscr{P}_{f}(\mathscr{C})[$ resp. on $\mathscr{P}(\mathscr{C})]$ defined by $\sigma_{X}(Y)=(-1)^{|X \cap Y|}$.

With the lattice $\mathscr{L}$ and the bonds $\mathscr{B}$ we associate the following subgroups $\mathscr{K}$ and $\Gamma$ of $\mathscr{P}(\mathscr{B})$

$$
\begin{aligned}
\mathscr{K} & =\{\beta \subset \mathscr{B} ; \forall x \in \mathscr{L}, \exists \text { an even number of } B \text { in } \beta \text { containing } x\} \\
\Gamma & =\{\gamma=\gamma(X) \subset \mathscr{B} ; \forall B \in \gamma \quad|B \cap X|=\text { odd }\}
\end{aligned}
$$

together with the following subgroups $\mathscr{S}$ and $\overline{\mathscr{B}}$ of $\mathscr{P}(\mathscr{L})$

$$
\begin{aligned}
\mathscr{S} & =\left\{S \subset \mathscr{L} ; \sigma_{S}(B)=+1 \forall B \in \mathscr{B}\right\} \\
\overline{\mathscr{B}} & =\left\{\bar{B} \subset \mathscr{L} ; \sigma_{S}(\bar{B})=+1 \forall S \in \mathscr{S},|\bar{B}|<\infty\right\} .
\end{aligned}
$$

Moreover, we denote by $\mathscr{K}_{f}$ the subgroup defined by $\mathscr{K}_{f}=\mathscr{K} \cap \mathscr{P}_{f}(\mathscr{B})$ and by $\Gamma^{(f)}$ the subgroup of $\Gamma$ defined by those $\gamma(X)$ with $|X|<\infty$.

The interest of these groups for duality and phase transition have been previously discussed [5-7].

A state $\omega$ is by definition a positive, linear, normalized form on $\mathfrak{A}$, the algebra of continuous functions on $\mathscr{P}(\mathscr{L})$; moreover $\omega$ is an equilibrium state for $\{\mathscr{L}, \mathscr{B}, K\}$ if 
for every $\Lambda \in \mathscr{P}_{f}(\mathscr{L})$ there exists a probability measure $\tilde{\omega}_{\Lambda}$ on $\mathscr{P}(\mathscr{L} / \Lambda)$ such that:

$$
\omega[A]=\int_{\mathscr{P}(\mathscr{\mathscr { C }} / \boldsymbol{A})} \tilde{\omega}_{\Lambda}(d Y) \sum_{X \subset \Lambda} A(X) \frac{\exp \left[-2 \sum_{B \in \gamma(X)} K(B) \sigma_{B}(Y)\right]}{\sum_{X \subset A} \exp \left[-2 \sum_{B \in \gamma(\bar{X})} K(B) \sigma_{B}(Y)\right]}
$$

for all $A$ in $\mathfrak{A}_{\Lambda}=\{A \in \mathfrak{A} ; A(X)=A(X \cap \Lambda) \forall X \subset \mathscr{L}\}$.

In the following discussion we shall restrict ourselves to the case of finite range potential only.

\section{Symmetric States and Symmetric Algebra}

A state $\omega$ is said "symmetric" if it is invariant under the internal symmetry group $\mathscr{S}$, i.e. for all $S$ in $\mathscr{S}$

$$
\omega[A]=\omega\left[\tau_{S} A\right] \text { where }\left(\tau_{S} A\right)(X)=A(S \cdot X) .
$$

A symmetric state is thus a positive, linear normalized form on the "symmetric algebra $\mathfrak{I}^{\text {sym }}$, where:

$$
\mathfrak{H}^{\text {sym }}=\left\{A \in \mathfrak{A} ; \tau_{\mathrm{S}} A=A \forall S \in \mathscr{S}\right\} .
$$

This symmetric algebra $\mathfrak{Q}^{\text {sym }}$ can be defined as the closure of the linear span of any of the following families of observables:

$$
\begin{array}{llll}
\text { 1) }\left\{\sigma_{\beta}\right\}_{\beta \in \mathscr{P}_{f}(\mathscr{B})} & \text { with } & \sigma_{\beta}=\prod_{B \in \beta} \sigma_{B} & \sigma_{\Phi}=1 \\
\text { 2) }\left\{\mu_{\beta}\right\}_{\beta \in \mathscr{P}_{f}(\mathscr{B})} & \text { with } & \mu_{\beta}=\prod_{B \in \beta} e^{-2 K(B) \sigma_{B}} & \mu_{\Phi}=1
\end{array}
$$

which have the following properties:

$$
\begin{aligned}
\sigma_{\beta_{1} \cdot \beta_{2}} & =\sigma_{\beta_{1}} \cdot \sigma_{\beta_{2}}, \sigma_{\beta \cdot \varkappa}=\sigma_{\beta} \text { for all } x \in \mathscr{K}_{f} \\
\mu_{\beta} & =\prod_{B \in \beta}\left[\operatorname{ch} 2 K(B)-\operatorname{sh} 2 K(B) \sigma_{B}\right] \\
\sigma_{\beta} & =\prod_{B \in \beta}\left[\operatorname{ch} 2 K_{*}(B)-\operatorname{sh} 2 K_{*}(B) \sigma_{B}\right] \quad \text { where } e^{-2 K_{*}(B)}=\operatorname{th} K(B) .
\end{aligned}
$$

In conclusion, any symmetric state $\omega$ will be uniquely defined by any one of the functions $\sigma(\beta)=\omega\left[\sigma_{\beta}\right]$ or $\mu(\beta)=\omega\left[\mu_{\beta}\right]$ on $\mathscr{P}_{f}(\mathscr{B})$, functions which are related by

$$
\mu=D_{K} \sigma \quad \sigma=D_{K_{*}} \mu
$$

and for any function $\varphi$ on $\mathscr{P}_{f}(\mathscr{B})$

$$
\left(D_{K} \varphi\right)(\beta)=\prod_{B \in \beta} \operatorname{ch} 2 K(B) \sum_{\bar{\beta} \subset \beta} \prod_{B \in \bar{\beta}} \operatorname{th}(-2 K(B)) \varphi(\bar{\beta}) .
$$

Proposition 1. The following statements are equivalent

1) $\omega$ is a symmetric equilibrium state.

2) The function $\sigma$ on $\mathscr{P}_{f}(\mathscr{B})$ is a solution of

$$
\text { i) } \sigma(\emptyset)=1, \sigma(\beta)=\sigma(\beta \cdot x) \quad \text { for all } \quad \beta \in \mathscr{P}_{f}(\mathscr{B}), \quad x \in \mathscr{K}_{f}
$$


ii) $\omega\left[\sigma_{\beta}\right]=\omega\left[\sigma_{\beta}\right.$ th $\left.\sum_{B \in \gamma} K(B) \sigma_{B}\right] \begin{aligned} & \text { for all } \beta \in \mathscr{P}_{f}(\mathscr{B}) \text { and } \gamma \in \Gamma^{(f)} \\ & \text { such that }|\beta \cap \gamma| \text { is odd }\end{aligned}$

defining a positive form $\omega$.

3) The function $\mu$ on $\mathscr{P}_{f}(\mathscr{B})$ is a solution of

i) $\mu(\emptyset)=1, \quad\left(D_{K_{*}} \mu\right)(\beta)=\left(D_{K_{*}} \mu\right)(\beta \cdot x) \quad$ for all $\quad \beta \in \mathscr{P}_{f}(\mathscr{B}), \quad x \in \mathscr{K}_{f}$

ii) $\mu(\beta)=\mu(\beta \cdot \gamma) \quad$ for all $\quad \beta \in \mathscr{P}_{f}(\mathscr{B}), \quad \gamma \in \Gamma^{(f)}$

defining a positive form $\omega$.

Let us note that it follows from the Equations i) of (3.1) [resp. (3.2)] that the function $\sigma$ (resp. $\mu$ ) defines a normalized, linear form $\omega$ on $\mathfrak{A}^{\text {sym }}$ which gives the meaning of the Equation ii). The proof of this proposition follows by direct computation from the equivalence previously shown in $[8,9]$. We remark moreover that in Equations (3.1) and (3.2), we could just as well consider only those $\gamma$ of the form $\gamma(x)$; any solution of this reduced set of equations will also be a solution of the full set of equations.

\section{Symmetric States and Duality}

Let $\{\mathscr{L}, \mathscr{B}, K\}$ be a general spin $\frac{1}{2}$ lattice system satisfying the condition $\Gamma^{(f)} \equiv \Gamma \cap \mathscr{P}_{f}(\mathscr{B})$ and let $\left\{\mathscr{L}^{*}, \mathscr{B}^{*}, K^{*}\right\}$ by any HT-LT dual for $\{\mathscr{L}, \mathscr{B}, K\}$ defined by means of a bijection $d: B \mapsto B^{*}$ of $\mathscr{B}$ onto $\mathscr{B}^{*}$ such that $K^{*}=K_{*} \circ d^{-1}$, which induces a bijection of $\mathscr{K}_{f}$ onto $\Gamma^{*} \cap \mathscr{P}_{f}\left(\mathscr{B}^{*}\right)[5]$.

It follows from a general argument on duality theory [5] that $d$ induces an isomorphism from $\mathscr{K}_{f}$ onto $\Gamma^{*} \cap \mathscr{P}_{f}\left(\mathscr{B}^{*}\right)$; moreover the conditions that $d$ is a bijection together with the fact that $\Gamma^{(f)} \equiv \Gamma \cap \mathscr{P}_{f}(\mathscr{B})$ implies that $d$ induces also an isomorphism from $\Gamma^{(f)}$ onto $\mathscr{K}_{f}^{*}$; indeed $\Gamma^{(f)} \equiv \Gamma \cap \mathscr{P}_{f}(\mathscr{B})$ iff Closure $\left(\mathscr{K}_{f}\right)=\mathscr{K}$ and therefore $d$ induces an isomorphism from $\mathscr{K}$ onto $\Gamma^{*} ;$ using the relations $\mathscr{K}^{\perp}=\Gamma^{(f)}$, $\Gamma^{* \perp}=\mathscr{K}_{f}^{*}$ concludes the argument.

Proposition 2. With any equilibrium state $\omega$ of $\{\mathscr{L}, \mathscr{B}, K\}$, we can associate a normalized linear form $\omega^{*}$ on $\mathfrak{H}^{* \mathrm{sym}}$ defined by:

$$
\omega^{*}\left[\sigma_{\beta^{*}}\right]=\omega\left[\mu_{d^{-1} \beta^{*}}\right]
$$

which is a solution of the Equilibrium Equation of Proposition 1. Moreover the mapping $\omega \mapsto \omega^{*}$ is injective and satisfies:

$$
\omega^{*}\left[\mu_{\beta^{*}}\right]=\omega\left[\sigma_{d^{-1} \beta^{*}}\right] \text {. }
$$

The proof of this proposition follows immediately from Proposition 1 and we omit the details of it.

The next problem one would like to investigate is under what conditions does $\omega$ yield an equilibrium state $\omega^{*}$ for the dual $\left\{\mathscr{L}^{*}, \mathscr{B}^{*}, K^{*}\right\}$, i.e. under what condition will $\omega^{*}$ be a positive form on $\mathfrak{U}^{* \text { sym }}$.

A linear form $\omega$ on $\mathfrak{U}^{\text {sym }}$ is positive if and only if, for any $\beta \in \mathscr{P}_{f}(\mathscr{B})$ and $\beta_{1} \in \Gamma^{(f)}$,

$$
\omega\left[\prod_{B_{1} \in \beta \cap \beta_{1}}\left(1-\sigma_{B_{1}}\right) \prod_{\substack{B \in \beta \\ B \notin \beta_{1}}}\left(1+\sigma_{B}\right)\right] \geqq 0 .
$$


By using this property, we conclude that $\omega^{*}$ is a positive form on $\mathfrak{U}^{* \text { sym }}$ if and only if for all $\beta \in \mathscr{P}_{f}(\mathscr{B})$ and $x \in \mathscr{K}_{f}$

$$
\omega\left[\prod_{B_{1} \in \beta \cap x}\left(1-\mu_{B_{1}}\right) \prod_{\substack{B \in \beta \\ B \notin \varkappa}}\left(1+\mu_{B}\right)\right] \geqq 0 .
$$

Using then the relations:

$$
\begin{aligned}
& 1-\mu_{B}=2 \operatorname{sh} K(B) e^{-K(B) \sigma_{B}} \\
& 1+\mu_{B}=2 \operatorname{ch} K(B) e^{-K(B) \sigma_{B}}
\end{aligned}
$$

we obtain the following result:

Lemma 1. The linear form $\omega^{*}$ associated with the symmetric equilibrium state $\omega$ of $\{\mathscr{L}, \mathscr{B}, K\}$ defines a symmetric equilibrium state for $\left\{\mathscr{L}^{*}, \mathscr{B}^{*}, K^{*}\right\}$ if and only if for any $\beta \in \mathscr{P}_{f}(\mathscr{B})$ and any $x \in \mathscr{K}_{f}$ we have:

$$
\left(\prod_{B_{1} \in \beta \cap x} \operatorname{sh} K\left(B_{1}\right)\right) \omega\left[\prod_{B \in \beta} e^{-K(B) \sigma_{B}} \prod_{B_{1} \in \beta \cap x} \sigma_{B_{1}}\right] \geqq 0 .
$$

In conclusion the set of symmetric equilibrium states satisfying the condition of Lemma 1 is precisely the set of equilibrium states to which duality can be applied.

In particular it follows immediately from GKS inequalities that for ferromagnetic systems,

- the state $\omega_{f}$ defined by means of "free boundary conditions" satisfies the condition of Lemma 1.

- the state $\omega_{+}$defined as the restriction to $\mathfrak{A}^{\text {sym }}$ of the state obtained by means of "+ boundary conditions" satisfies the condition of Lemma 1.

Therefore, $\omega_{f}$ and $\omega_{+}$yield by duality symmetric equilibrium states of the dual; moreover, we have the following result:

Theorem 1. Let $\{\mathscr{L}, \mathscr{B}, K\}$ be a ferromagnetic system such that ${ }^{2}$

i) $\Gamma^{(f)} \equiv \Gamma \cap \mathscr{P}_{f}(\mathscr{B})$

ii) there exists a sequence of finite volume $\Lambda_{i} \rightarrow \mathscr{L}$ such that for any $\Lambda_{i}$ and $|X|<\infty$ the conditions $\sigma_{B}(X)=+1 \forall B \subset \mathscr{L} / \Lambda_{i}$ imply $X=Y S_{f}$ with $Y \subset \Lambda_{i}$ and $S_{f} \in \mathscr{S} \cap \mathscr{P}_{f}(\mathscr{L})^{3}$ then

1) for any HT-LT dual $\left\{\mathscr{L}^{*}, \mathscr{B}^{*}, K^{*}\right\}$ defined by means of a bijection $d: \mathscr{B} \rightarrow \mathscr{B}^{*}$ we have:

$\left(\omega_{+}\right)^{*}=\omega_{f}^{*}$.

2) If moreover the dual system satisfies the same condition then

$\left(\omega_{f}\right)^{*}=\omega_{+}^{*}$

and any equilibrium state $\omega$ for $\{\mathscr{L}, \mathscr{B}, K\}$ which yields by duality a state $\omega^{*}$ for $\left\{\mathscr{L}^{*}, \mathscr{B}^{*}, K^{*}\right\}$ satisfies the inequality:

$$
\begin{aligned}
\omega_{+}\left[\sigma_{\beta}\right] \geqq \omega\left[\sigma_{\beta}\right] \geqq \omega_{f}\left[\sigma_{\beta}\right] \geqq \prod_{B \in \beta} \operatorname{th} K(B) \\
1 \geqq \omega_{f}\left[\mu_{\beta}\right] \geqq \omega\left[\mu_{\beta}\right] \geqq \omega_{+}\left[\mu_{\beta}\right] .
\end{aligned}
$$

2 Remark that these are exactly the conditions which are needed for the Peierls argument $[6,10]$

3 It is expected that for $\mathbb{Z}^{v}$-invariant systems, this condition is always satisfied 
Proof. A finite subsystem $\left\{\Lambda, \mathscr{B}^{+}(\Lambda), K\right\}$ [resp. $\left.\left\{\Lambda, \mathscr{B}^{f}(\Lambda), K\right\}\right]$ of $\{\mathscr{L}, \mathscr{B}, K\}$ with "+" boundary conditions [resp. "free" boundary conditions] is defined by $\Lambda \in \mathscr{P}_{f}(\mathscr{L})$ and the corresponding set of bonds

$$
\begin{aligned}
& \mathscr{B}^{+}(\Lambda)=\{B \in \mathscr{B} ; \Lambda \cap B \neq \emptyset\} \\
& \mathscr{B}^{f}(\Lambda)=\{B \in \mathscr{B} ; B \subset \Lambda\} .
\end{aligned}
$$

Let $\{\mathscr{L}, \mathscr{B}, K\}$ be a ferromagnetic system satisfying the conditions i) and ii) of Theorem 1 . For any $\Lambda_{i}$, condition ii) implies

$$
\Gamma\left(\Lambda_{i}\right)=\left\{\gamma(X) ; X \subset \Lambda_{i}\right\} \equiv \Gamma^{(f)} \cap \mathscr{P}\left(\mathscr{B}^{+}\left(\Lambda_{i}\right)\right)
$$

and we have

$$
d \Gamma\left(\Lambda_{i}\right)=\mathscr{K}_{f}^{*} \cap \mathscr{P}\left(d \mathscr{B}^{+}\left(\Lambda_{i}\right)\right) .
$$

Defining $\Lambda_{i}^{*}=\bigcup_{B \in \mathscr{B}^{+}\left(\Lambda_{i}\right)} B^{*}$ the system $\left\{\Lambda_{i}^{*}, d \mathscr{B}^{*}\left(\Lambda_{i}\right), K^{*}\right\}$ is a HT-LT dual for $\left\{\Lambda_{i}, \mathscr{B}^{*}\left(\Lambda_{i}\right), K\right\} ;$ therefore [11]

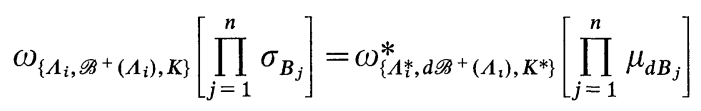

where $B_{j} \in \mathscr{B}^{+}\left(\Lambda_{i}\right) j=1, \ldots, n$ and $\omega_{\left\{\Lambda_{i}, \mathscr{B}^{+}\left(\boldsymbol{A}_{i}\right), K\right\}} \quad\left[\right.$ resp. $\left.\omega_{\left\{\Lambda_{i}^{*}, d \mathscr{B}^{+}\left(\boldsymbol{\Lambda}_{\imath}\right), K^{*}\right\}}^{*}\right]$ denotes the Gibbs states of the finite systems $\left\{\Lambda_{i}, \mathscr{B}^{+}\left(\Lambda_{i}\right), K\right\} \quad\left[\operatorname{resp} .\left\{\Lambda_{i}^{*}, d \mathscr{B}^{+}\left(\Lambda_{i}\right), K^{*}\right\}\right]$.

Let $\tilde{\Lambda}_{i}^{*} \subset \Lambda_{i}^{*}$ be a largest volume such that $\mathscr{B}^{f}\left(\tilde{\Lambda}_{i}^{*}\right) \subset d \mathscr{B}^{+}\left(\Lambda_{i}\right)$. Since $\left\{\Lambda_{i}^{*}, d \mathscr{B}^{+}\left(\Lambda_{i}\right)\right.$, $\left.K^{*}\right\}$ is a ferromagnetic system with $d \mathscr{B}^{+}\left(\Lambda_{i}\right) \subset \mathscr{B}^{* f}\left(\Lambda_{i}^{*}\right)$ it follows from Griffiths inequalities that for any $\beta^{*} \in \mathscr{P}\left(\mathscr{B}^{f}\left(\tilde{\Lambda}_{i}^{*}\right)\right)$

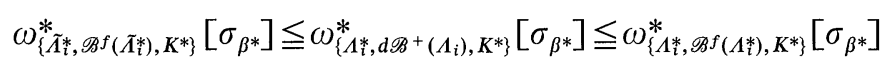

but $\Lambda_{i} \rightarrow \mathscr{L}$ implies $\Lambda_{i}^{*} \rightarrow \mathscr{L}^{*}$ and $\tilde{\Lambda}_{i}^{*} \rightarrow \mathscr{L}^{*}$; therefore

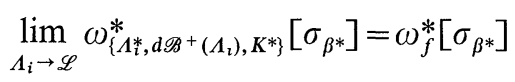

and inally for any $\beta^{*} \in \mathscr{P}_{f}\left(\mathscr{B}^{*}\right)$, we have

$$
\begin{array}{r}
\left(\omega_{+}\right)^{*}\left[\mu_{\beta^{*}}\right] \omega_{+}\left[\sigma_{d^{-1} \beta^{*}}\right]=\lim _{\Lambda_{\imath} \rightarrow \mathscr{L}} \omega_{\left\{\Lambda_{l}, \mathscr{B}^{+}\left(\lambda_{i}\right), K\right\}}\left[\sigma_{d^{-1} \beta^{*}}\right] \\
=\lim _{\Lambda_{i}^{*} \rightarrow \mathscr{L}^{*}} \omega_{\left\{\Lambda_{i}^{*}, d \mathscr{B}^{+}\left(\Lambda_{i}\right), K^{*}\right\}}^{*}\left[\mu_{\beta^{*}}\right]=\omega_{f}^{*}\left[\mu_{\beta^{*}}\right]
\end{array}
$$

i.e.

$$
\left(\omega_{+}\right)^{*}=\omega_{f}^{*} .
$$

Moreover if the dual system also satisfies the conditions i) and ii) of Theorem 1 , the above result reads

$$
\left(\omega_{+}^{*}\right)^{*}=\omega_{f}^{* *}=\omega_{f}
$$

which yields, since $\left(\omega_{f}\right)^{*}$ is a state, $\left(\omega_{f}\right)^{*}=\omega_{+}^{*}$. 
Now let $\omega=\lim _{\Lambda \rightarrow \mathscr{L}} \dot{\omega}_{\Lambda, Y}$ where $\omega_{\Lambda, Y}$ is the Gibbs state for the system defined on $\Lambda$ with boundary condition $Y$, then

$$
\begin{aligned}
& \left(\omega_{\Lambda, Y}\right)^{*}\left[\sigma_{\beta^{*}}\right]=\omega_{\Lambda, Y}\left[\mu_{\beta}\right] \\
& \left(\omega_{\Lambda, Y}\right)^{*}\left[\sigma_{\beta^{*}}\right]=\frac{\left\langle\sigma_{\beta^{*}} \sigma_{b^{*}}\right\rangle_{\Lambda^{*}, f}}{\left\langle\sigma_{b^{*}}\right\rangle_{\Lambda^{*}, f}} \geqq\left\langle\sigma_{\beta^{*}}\right\rangle_{\Lambda^{*}, f}
\end{aligned}
$$

where $A^{*}=\bigcup_{B \cap A \neq \Phi} B^{*}$ and $b^{*}=\left\{d B ; \sigma_{B}(Y)=-1\right.$ if $\left.B \cap \Lambda \notin\{\emptyset, B\}\right\}$ therefore:

$$
\omega\left[\mu_{\beta}\right] \geqq \omega_{f}^{*}\left[\sigma_{\beta^{*}}\right]=\omega_{+}\left[\mu_{\beta}\right]
$$

and for any equilibrium state $\omega$ of the ferromagnetic system $\{\mathscr{L}, \mathscr{B}, K\}$ we have:

$$
\omega\left[\mu_{\beta}\right] \geqq \omega_{+}\left[\mu_{\beta}\right] .
$$

If moreover $\omega^{*}$ is an equilibrium state of $\left\{\mathscr{L}^{*}, \mathscr{B}^{*}, K^{*}\right\}$, we must have

$$
\omega^{*}\left[\mu_{\beta^{*}}\right] \geqq \omega_{+}^{*}\left[\mu_{\beta^{*}}\right]
$$

which yields :

$$
\omega\left[\sigma_{\beta}\right] \geqq \omega_{f}\left[\sigma_{\beta}\right] .
$$

An important class of states which yield by duality states of the dual systems is the class of translation invariant states which we now discuss.

Theorem 2. Let $\{\mathscr{L}, \mathscr{B}, K\}$ be a $\mathbb{Z}^{v}$-invariant, ferromagnetic, lattice system satisfying the conditions of Theorem 1 and let $\omega$ be any $\mathbb{Z}^{v}$-invariant equilibrium state. With $\left\{\mathscr{L}^{*}, \mathscr{B}^{*}, K^{*}\right\}$ a HT-LT dual which is $\mathbb{Z}^{v}$-invariant and such that the bijection $d: \mathscr{B} \rightarrow \mathscr{B}^{*}$ commutes with translations, then the linear form $\omega^{*}$ associated to $\omega$ by duality is a symmetric, $\mathbb{Z}^{v}$-invariant, equilibrium state for the dual system.

The proof of this theorem is a generalization of the technique used in [1] and gives in fact the natural setting for the result established there.

Proof. By definition $\{\mathscr{L}, \mathscr{B}, K\}$ is $\mathbb{Z}^{v}$-invariant, if $\mathbb{Z}^{v}$ acts as a group of translations of $\mathscr{L}$ such that $a \in \mathbb{Z}^{v}$ and $B \in \mathscr{B}$ imply $B+a \in \mathscr{B}$ with $K(B+a)=K(B)$; moreover $\omega$ is said $\mathbb{Z}^{v}$-invariant if $\omega\left[\sigma_{X+a}\right]=\omega\left[\sigma_{X}\right]$ for any $a \in \mathbb{Z}^{v}, X \in \mathscr{P}_{f}(\mathscr{L})$.

For the sequence of volumes $\Lambda_{i} \rightarrow \mathscr{L}$, we consider the unperturbed finite systems $\left\{\Lambda_{i}, \mathscr{B}^{+}\left(\Lambda_{i}\right), K\right\}$, together with the HT-LT duals $\left\{\Lambda_{i}^{*}, d \mathscr{B}^{+}\left(\Lambda_{i}\right), K^{*}\right\}$.

With $\beta$ an element in $\mathscr{P}_{f}(\mathscr{B})$ we associate the systems $\Sigma_{i}=\left\{\Lambda_{i}, \mathscr{B}_{i}, K_{i}\right\}, \tilde{\Sigma}_{i}^{*}=\left\{\Lambda_{i}^{*}\right.$, $\left.\tilde{\mathscr{B}}_{i}^{*}, \tilde{K}_{i}^{*}\right\}$ defined respectively by the perturbed Hamiltonians :

$$
\begin{aligned}
& H_{\Lambda_{\imath}, \lambda}=-\sum_{B \in \mathscr{B}^{+}\left(\Lambda_{\imath}\right)} K(B) \sigma_{B}-\lambda \sum_{a \in M_{\imath}} \sigma_{\beta+a}=H_{\Lambda_{i},+}-\lambda \delta H_{\Lambda_{\imath}} \\
& \tilde{H}_{\Lambda_{i}^{*}, \lambda}=-\sum_{B^{*} \in d \mathscr{B}^{+}\left(\Lambda_{i}\right)} K^{*}\left(B^{*}\right) \sigma_{B^{*}}-\lambda \sum_{a \in M_{i}} \sigma_{\beta^{*}+a}=H_{\Lambda_{i}^{*}}^{*}-\lambda \delta H_{\Lambda_{\imath}^{*}}^{*}
\end{aligned}
$$

where $M_{i}$ is the set of those $a \in \mathbb{Z}^{v}$ such that $B+a \subset \Lambda_{i}$ for all $B$ in $\beta$.

We denote by $Z_{i}(K, \lambda), \tilde{Z}_{i}\left(K^{*}, \lambda\right)$ and $p(K, \lambda), \tilde{p}\left(K^{*}, \lambda\right)$ the partition functions and the infinite volume free energies associated with the systems $\Sigma_{i}$ and $\tilde{\Sigma}_{i}^{*}$.

The system $\tilde{\Sigma}_{i}^{*}$ is not the dual of $\Sigma_{i}$ except for $\lambda=0$ in which case both systems reduce to the original unperturbed systems. We see however from its definition that 
the derivative of $\tilde{p}\left(K^{*}, \lambda\right)$ at $\lambda=0$ formally gives the dual expression of the average correlation function $\omega\left[\sigma_{\beta}\right]$ (up to a multiplicative constant). The proof of the theorem consists then in establishing that $\tilde{p}\left(K^{*}, \lambda\right)$ approaches in a suitable way the free energy of the infinite system associated with the dual of $\Sigma_{i}$.

We have:

$$
\begin{aligned}
Z_{i}(K, \lambda) & =\sum_{X \subset \Lambda_{i}} \exp \left[-H_{\Lambda_{i},+}(X)\right] \prod_{a \in M_{i}} e^{\lambda_{\beta+a}(X)} \\
& =(\operatorname{ch} \lambda)^{\left|M_{i}\right|} \sum_{X \subset \Lambda_{i}} \exp \left[-H_{\Lambda_{i},+}(X)\right] \sum_{S \subset M_{i}}(\operatorname{th} \lambda)^{|S|} \sigma_{\beta[S]}(X)
\end{aligned}
$$

where $\beta[S]=\prod_{a \in S}(\beta+a)$ the product being taken in $\mathscr{P}(\mathscr{B})$.

Applying the duality transformations [11] we obtain:

$$
\begin{aligned}
& Z_{i}(K, \lambda)=(\operatorname{ch} \lambda)^{\left|M_{i}\right|} \frac{Z_{i}(K, \lambda=0)}{\tilde{Z}_{i}\left(K^{*}, \lambda=0\right)} \sum_{X^{*} \mathcal{C} \Lambda_{i}^{*}} \exp \left[-H_{\Lambda_{i}^{*}}^{*}\left(X^{*}\right)\right] \\
& \cdot \sum_{S C M_{i}}(\operatorname{th} \lambda)^{|S|} \exp -2 \sum_{B^{*} \in \beta[S]^{*}} K^{*}\left(B^{*}\right) \sigma_{B^{*}}\left(X^{*}\right) .
\end{aligned}
$$

By taking into account the identity,

$$
-2 \sum_{B^{*} \in \beta[S]^{*}} K^{*}\left(B^{*}\right) \sigma_{B^{*}}=\sum_{\Phi \neq R \subset C}(-2)^{|R|} \sum_{B^{*} \in \bigcap_{a \in R}\left(\beta^{*}+a\right)} K^{*}\left(B^{*}\right) \sigma_{B^{*}}
$$

we can write

$$
Z_{i}(K, \lambda)=(\operatorname{ch} \lambda)^{\left|M_{i}\right|} \frac{Z_{i}(K, \lambda=0)}{\tilde{Z}_{i}\left(K^{*}, \lambda=0\right)} \sum_{X^{*} \Lambda_{i}^{*}} \exp \left[-H_{\Lambda_{i}^{*}}^{*}\left(X^{*}\right)+\log Z_{M_{i}}\left(\Phi_{X^{*}} \text { th } \lambda\right)\right]
$$

where $Z_{M_{i}}\left(\Phi_{X^{*}}\right.$, th $\left.\lambda\right)$ is the partition function of the system $\left\{M_{i}, \Phi_{X^{*}}\right.$, th $\left.\lambda\right\}$ defined in the lattice gas language by $M_{i} \subset \mathbb{Z}^{v}$, the activity th $\lambda$ and the interaction potential $\Phi_{X^{*}}$ given by:

$$
\begin{aligned}
& \Phi_{X^{*}}(R)=(-2)^{|R|} \sum_{B^{*} \in \bigcap_{a \in R}\left(\beta^{*+a}\right)} K^{*}\left(B^{*}\right) \sigma_{B^{*}}\left(X^{*}\right) \\
& \Phi_{X^{*}}(\emptyset)=0 .
\end{aligned}
$$

We thus obtain from Equation (4.3) and the definitions introduced:

$$
\begin{aligned}
& \frac{\left|\Lambda_{i}\right|}{\left|M_{i}\right|}\left[p_{\Lambda_{i}}(K, \lambda)-p_{\Lambda_{i}}(K, 0)\right]-\frac{\left|\Lambda_{i}^{*}\right|}{\left|M_{i}\right|}\left[\tilde{p}_{\Lambda_{i}^{*}}\left(K^{*}, \lambda\right)-\tilde{p}_{\Lambda_{i}}\left(K^{*}, 0\right)\right] \\
& =\ln (\operatorname{ch} \lambda)+\frac{1}{\left|M_{i}\right|} \ln \frac{A_{i}}{B_{i}}
\end{aligned}
$$

with

$$
\begin{aligned}
& A_{i}=\sum_{X^{*} \subset A_{i}^{*}} \exp \left[-H_{\Lambda_{i}^{*}}^{*}\left(X^{*}\right)+\log Z_{M_{i}}\left(\Phi_{X^{*}}, \text { th } \lambda\right)\right] \\
& B_{i}=\sum_{X^{*} \subset \Lambda_{i}^{*}} \exp \left[-H_{A_{i}^{*}}^{*}\left(X^{*}\right)+\lambda \sum_{a \in M_{i}} \mu_{\beta^{*}+a}\left(X^{*}\right)\right] .
\end{aligned}
$$


The potential $\Phi_{X^{*}}$, function on $\mathscr{P}_{f}\left(\mathbb{Z}^{\nu}\right)$, depends on the configuration $X^{*} \in \mathscr{P}_{f}\left(\mathscr{L}^{*}\right)$ and is not translation invariant; however, it follows from Equation (4.4) that $\Phi_{X^{*}}$ is a finite range potential and that the norm $\left\|\Phi_{X^{*}}\right\|$ which is defined by

$$
\left\|\Phi_{X^{*}}\right\|=\sup _{a \in \mathbb{Z}^{v}} \sum_{R \ni a}\left|\Phi_{X^{*}}(R)\right|
$$

can be uniformly bounded independently of $X^{*}$. This allows us to use the low activity properties of the system $\left\{M_{i}, \Phi_{X^{*}}\right.$, th $\left.\lambda\right\}$ in order to study the behaviour of $Z_{M_{i}}\left(\Phi_{X^{*}}\right.$, th $\left.\lambda\right)$. The basic results needed for this study are those of [12].

The arguments to continue the proof do not differ essentially from those employed in the proof of the theorem in [1] and we will not repeat them here. The main point is to notice that

$$
\log Z_{M_{i}}\left(\Phi_{X^{*}}, \operatorname{th} \lambda\right)=\sum_{a \in M_{i}} \int_{0}^{\operatorname{th} \lambda} \frac{d t}{t} \omega_{\left\{M_{i}, \Phi_{X *, t}\right.}\left[\sigma_{a}\right]
$$

and to use the low activity expansion

$$
\omega_{\left\{\mathbb{Z}^{v}, \Phi_{\left.X^{*}, t\right\}}\right.}\left[\sigma_{a}\right]=t \mu_{\beta^{*}+a}\left(X^{*}\right)+R_{2}(t)
$$

to control the difference between the terms $\log Z_{M_{\imath}}\left(\Phi_{X^{*}}\right.$, th $\left.\lambda\right)$ and $\lambda \sum_{a \in M_{i}} \mu_{\beta^{*}+a}\left(X^{*}\right)$.

As a consequence, one obtains that:

$$
\left|\frac{\alpha}{\lambda}\{p(K, \lambda)-p(K, 0)\}-\frac{\alpha^{*}}{\lambda}\left\{\tilde{p}\left(K^{*}, \lambda\right)-\tilde{p}\left(K^{*}, 0\right)\right\}\right| \leqq \varepsilon(|\lambda|)
$$

where $\varepsilon(|\lambda|) \rightarrow 0$ as $|\lambda| \rightarrow 0$, and $\alpha=\left|\mathscr{L} / \mathbb{Z}^{v}\right|, \alpha^{*}=\left|\mathscr{L}^{*} / \mathbb{Z}^{v}\right|$.

Since $p(K, \lambda)$ and $\tilde{p}\left(K^{*}, \lambda\right)$ are convex functions of $\lambda$, the right and left derivatives of these functions exist and from the formula above, we deduce that:

$$
\left.\alpha \frac{\partial p(K, \lambda)}{\partial \lambda}\right|_{\lambda=0^{-}}=\left.\alpha^{*} \frac{\partial \tilde{p}\left(K^{*}, \lambda\right)}{\partial \lambda}\right|_{\lambda=0^{-}} \leqq\left.\alpha \frac{\partial p(K, \lambda)}{\partial \lambda}\right|_{\lambda=0^{+}}=\left.\alpha^{*} \frac{\partial \tilde{p}\left(K^{*}, \lambda\right)}{\partial \lambda}\right|_{\lambda=0} .
$$

Therefore

$$
\left.\frac{\partial p(K, \lambda)}{\partial \lambda}\right|_{\lambda=0^{-}} \leqq \frac{1}{\alpha} \omega\left[\sigma_{\beta}\right] \leqq\left.\frac{\partial p(K, \lambda)}{\partial \lambda}\right|_{\lambda=0^{+}}
$$

implies that

$$
\left.\frac{\partial \tilde{p}\left(K^{*}, \lambda\right)}{\partial \lambda}\right|_{\lambda=0^{-}} \leqq \frac{1}{\alpha^{*}} \omega^{*}\left[\mu_{\beta^{*}}\right] \leqq\left.\frac{\partial \tilde{p}\left(K^{*}, \lambda\right)}{\partial \lambda}\right|_{\lambda=0^{+}} .
$$

But these conditions written for all $\beta \in \mathscr{P}_{f}(\mathscr{B})$ are just the conditions for the $\mathbb{Z}^{v}$ invariant linear forms $\frac{1}{\alpha} \omega$ and $\frac{1}{\alpha^{*}} \omega^{*}$ to respectively define tangent planes to the graph of the free energies $p(K, \lambda)$ and $\tilde{p}\left(K^{*}, \lambda\right)$ at $\lambda=0$. Moreover, it is known that these tangent planes are in one to one correspondence with the $\mathbb{Z}^{v}$-invariant equilibrium states of the systems $\{\mathscr{L}, \mathscr{B}, K\}$ and $\left\{\mathscr{L}^{*}, \mathscr{B}^{*}, K^{*}\right\}$ [13]. It should only be remarked that this last statement is derived in ref. [13] only for the case 
$\mathscr{L}=\mathbb{Z}^{v}$ which gives $\alpha=1$; however, this result extends to the more general case which we have considered up to the multiplicative factor $\alpha^{-1}$ which arises from the fact that $\lim _{\Lambda_{i} \rightarrow \mathscr{L}} \frac{\left|\Lambda_{i}\right|}{\left|M_{i}\right|}=\left|\mathscr{L} / \mathbb{Z}^{v}\right|$. This achieves the proof of the theorem.

Corollary. If $\{\mathscr{L}, \mathscr{B}, K\}$ is a $\mathbb{Z}^{v}$-invariant, ferromagnetic, lattice system satisfying conditons of Theorem 1 , then any $\mathbb{Z}^{v}$-invariant, symmetric equilibrium state $\omega$ satisfies the inequality

(i) $\omega_{+}\left[\sigma_{\beta}\right] \geqq \omega\left[\sigma_{\beta}\right] \geqq \omega_{f}\left[\sigma_{\beta}\right] \forall \beta \in \mathscr{P}_{f}(\mathscr{B})$.

(ii) The symmetric equilibrium state is unique at low temperature.

Part (i) follows immediately from Theorems 1 and 2.

Part (ii) is a consequence of Theorem 2 where we recover the known results concerning the unicity of symmetric Invariant Equilibrium State at Low Temperature. This follows from the unicity of the equilibrium state in the dual model at high temperature. In general the bounds given by means of duality improve those previously obtained by means of the Asano's contraction method.

Let us note that it is expected that this unicity property holds for all temperature. It thus follows from the above conclusions that it would be sufficient to show that "+" and "free" boundary conditons yield states which coincide on $\mathfrak{I}^{\text {sym }}$ to establish this conjecture. Such a result has been proved however only for the 2dimensional Ising model [2]; it is a consequence of the theorems above and the Lebowitz's results showing the unicity of the equilibrium state above the critical temperature [16].

\section{On the Surface Tension}

In this section we give an application of duality relations to the study of the surface tension. For simplicity, we shall restrict ourselves to the case of crystal lattice systems, i.e. $\mathscr{L}=\mathbb{Z}^{v}$.

The problem of the surface tension for the Ising model in a low temperature region has been extensively studied by Abraham, Gallavotti and Martin-Löf (see [14] and the references quoted there). They have, in particular, proved the equivalence of several difinitions which can be proposed for the surface tension. We shall choose here Fisher's definition [17] and our aim will be to prove the existence of the infinite volume surface tension. As we shall see, this result can be deduced rather easily from Griffith's inequalities and standard techniques in the study of the thermodynamic limit. By this methods the existence of the infinite volume surface tension can be proved at any value of the temperature.

Let $\left\{\mathbb{Z}^{v}, \mathscr{B}, \quad K\right\}$ be a lattice system and let us decompose $\mathbb{Z}^{v}$ into $\mathbb{Z}_{u}^{v} \cup \mathbb{Z}_{d}^{v}$ where $\mathbb{Z}_{u}^{v}$ stands for those $x \in \mathbb{Z}^{v}$ such that $x_{v}>0$. With $S$ in $\mathscr{S}^{4}$, we consider the finite system $\Lambda$ with boundary conditions $Y=S \cap \mathbb{Z}_{d}^{v}$ where $\Lambda$ is taken to be a parallelipiped with sides $\left(L_{1}, \ldots, L_{v-1}, 2 M\right)$ symmetric with respect to the plane $x_{v}=1 / 2$, and the boundary condition is such that $\sigma_{x}(Y)=+1$ if $x \in \mathbb{Z}_{u}^{v} \cap \Lambda^{c} \sigma_{x}(Y)=\sigma_{x}(S)$ if $x \in \mathbb{Z}_{d}^{v} \cap \Lambda^{c}$ and $\Lambda^{c}=\mathbb{Z}^{v} / \Lambda$ (see Fig. 1 ).

\footnotetext{
$4 \quad$ We recall that for ferromagnetic systems the elements of $\mathscr{S}$ are the "ground states" of the infinite system
} 


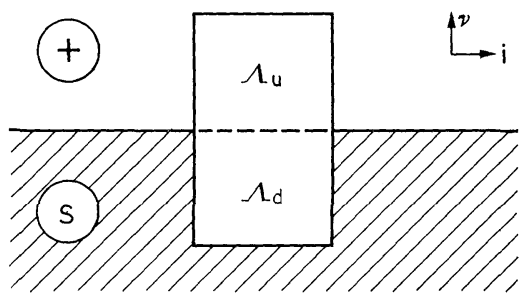

Fig.1. Finite system $\Lambda=\Lambda_{d} \cup \Lambda_{u}$ with boundary condition $Y=S \cap \mathbb{Z}_{d}^{v}$

The Hamiltonian $H_{\Lambda, Y}$ of the finite system $\Lambda$ with boundary conditon $Y$ outside of $\Lambda$ is given by:

$$
\begin{aligned}
H_{\Lambda, Y}(X) & =-\sum_{B \in \mathscr{B}^{+}(\Lambda)} K(B) \sigma_{B}\left(X \cdot Y_{\Lambda}\right) \\
& =H_{\Lambda,+}(X)+2 \sum_{B \in \mathcal{Y}\left(Y_{A}\right) \cap \mathscr{B}^{+}(\Lambda)} K(B) \sigma_{B}(X)
\end{aligned}
$$

where $Y_{\Lambda}=Y \cap \Lambda^{c}=S \cap \mathbb{Z}_{d}^{v} \cap \Lambda^{c}$.

We denote by $Z_{\Lambda}^{(+, S)}$ and $Z_{\Lambda}^{(+)}$the partition functions corresponding respectively to $(Y)$ and $(+)$ boundary condition.

Theorem 3. Let $\left\{\mathbb{Z}^{v}, \mathscr{B}, K\right\}$ be a ferromagnetic lattice system satisfying the conditions of Theorem 1; for any $S \in \mathscr{S}$ the following limit:

$$
\tau_{(+, S)}=\lim _{L_{1}, \ldots, L_{v-1} \rightarrow \infty} \lim _{M \rightarrow \infty} \frac{1}{L_{1} \ldots L_{v-1}} \log \frac{Z_{\Lambda}^{(+, S)}}{Z_{\Lambda}^{(+)}}
$$

exists and is called the "surface tension between the phases $(+)$ and $(S)$ ". Moreover $\tau_{(+, S)}$ is non-positive and bounded below.

Proof. It follows from the above definitions and the duality relation, that:

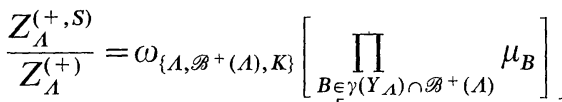

$$
\begin{aligned}
& \left.=\omega_{\left\{\Lambda^{*}, d \mathscr{B}^{+}(\Lambda), K^{*}\right\}}^{*} \prod_{B^{*} \in d\left(\gamma\left(Y_{A}\right) \cap \mathscr{B}^{+}(\Lambda)\right)}\right] \sigma_{B^{*}} .
\end{aligned}
$$

Using the identity $\left(S \cap \Lambda_{d}^{c}\right) \cdot Y_{\Lambda}=S \cap \mathbb{Z}_{u}^{v}$ with $\Lambda_{d}=\Lambda \cap \mathbb{Z}_{d}^{v}$ it follows that

$$
\gamma\left(Y_{\Lambda}\right) \cap \mathscr{B}^{+}(\Lambda)=\gamma\left(S \cap \Lambda_{d}^{c}\right) \cdot \beta_{\Lambda}
$$

where:

$$
\beta_{A}=\left\{B \in \mathscr{B}_{A}^{+} ; \sigma_{B}\left(S \cap \mathbb{Z}_{u}^{v}\right)=-1\right\}
$$

we thus have by definition of duality

$$
\frac{Z_{\Lambda}^{(+, S)}}{Z_{\Lambda}^{(+)}}=\omega_{\left\{\Lambda^{*}, d B^{+}(\Lambda), K^{*}\right\}}^{*}\left[\sigma_{\beta_{\Lambda}^{*}}\right]
$$

where $\beta_{\Lambda}$ is independent of $M$, for large $M$. [Since $\beta_{\Lambda}$ is a subset of bonds in $\mathscr{B}^{+}(\Lambda)$ with non empty intersection with the upper and lower half-space.] 


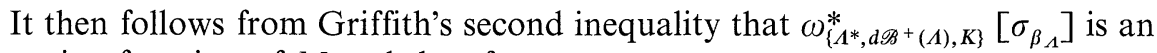
increasing function of $M$ and therefore

$$
\lim _{M \rightarrow \infty} \log \frac{Z_{\Lambda}^{(+, S)}}{Z_{\Lambda}^{(+)}}
$$

exists. On the other hand, it follows from the Corollary that

$$
1 \geqq \omega_{\left\{\Lambda^{*}, d \mathscr{B}^{+}(\Lambda), K^{*}\right\}}^{*}\left[\sigma_{\beta_{A}^{*}}\right] \geqq \prod_{B^{*} \in \beta_{\Lambda}} \operatorname{th} K^{*}\left(B^{*}\right)=\prod_{B \in \beta_{\Lambda}} e^{-2 K(B)} .
$$

Therefore, we have:

$$
0 \geqq \log \frac{Z^{(+, S)}}{Z^{(+)}} \geqq-2 \sum_{B \in \beta_{A}} K(B) \geqq-2 \bar{K} \cdot C \cdot \prod_{i=1}^{\nu-1} L_{i}
$$

where $\bar{K}=\sup _{B \in \mathscr{B}}|K(B)|$ and $C$ is some finite positive constant.

This shows that $\tau_{(+, S)}$, when it exists, in non positive and bounded below.

Next we introduce the parallelipipeds $\Lambda^{\prime}, \Lambda^{\prime \prime}$, and $\Lambda=\Lambda^{\prime} \cup \Lambda^{\prime \prime}$ of sides $\left(L_{1}, \ldots, L_{i}^{\prime}, \ldots, L_{v-1}, 2 M\right),\left(L_{1}, \ldots, L_{i}^{\prime \prime}, \ldots, L_{v-1}, 2 M\right)$ and $\left(L_{1}, \ldots, L_{i}^{\prime}+L_{i}^{\prime \prime}, \ldots, L_{v-1}, 2 M\right)$. We then have

$$
\beta_{\Lambda}=\beta_{\Lambda^{\prime}} \cdot \beta_{\Lambda^{\prime \prime}} \cdot \delta \beta_{\Lambda}
$$

where $\delta \beta_{\Lambda}$ is a subset of those bonds in $\mathscr{B}^{+}\left(\Lambda^{\prime}\right) \cap \mathscr{B}^{+}\left(\Lambda^{\prime \prime}\right)$ with non empty intersection with upper half-space and lower half-space.

Using again Griffiths' second inequality, we get:

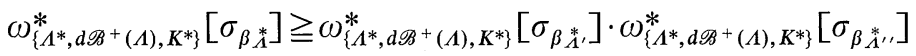

$$
\begin{aligned}
& \cdot \omega_{\left\{\Lambda^{*}, d \mathscr{B}^{+}(\Lambda), K^{*}\right\}}^{*}\left[\sigma_{\delta_{\beta_{\Lambda}^{*}}^{*}}\right] \\
& \geqq \omega_{\left\{\Lambda^{\prime *}, d \mathscr{B}^{+}\left(\Lambda^{\prime}\right), K^{*}\right\}}^{*}\left[\sigma_{\beta_{\Lambda^{\prime}}^{*}}\right] \cdot \omega_{\left\{\Lambda,,^{*}, d \mathscr{B}^{+}\left(\Lambda^{\prime \prime}\right), K^{*}\right\}}^{*}\left[\sigma_{\beta \Lambda^{* \prime \prime}}\right] \prod_{B \in \delta_{\beta_{A}}} e^{-2 K(B)} .
\end{aligned}
$$

Introducing the function

$$
f\left(L_{1}, \ldots, L_{v-1}\right)=\lim _{M \rightarrow \infty} \log \frac{Z_{\Lambda}^{(+, S)}}{Z_{\Lambda}^{(+)}}
$$

the above inequality gives:

$$
\begin{aligned}
& \quad f\left(L_{1}, \ldots, L_{i}^{\prime}+L_{i}^{\prime \prime}, \ldots, L_{v-1}\right) \geqq f\left(L_{1}, \ldots, L_{i}^{\prime}, \ldots, L_{v-1}\right)+f\left(L_{1}, \ldots, L_{i}^{\prime \prime}, \ldots, L_{v-1}\right) \\
& -2 \tilde{C} \bar{K} \frac{L_{1} \ldots L_{v-1}}{L_{i}} .
\end{aligned}
$$

Therefore the function

$$
g\left(L_{1}, \ldots, L_{v-1}\right)=f\left(L_{1}, \ldots, L_{v-1}\right)-2 \tilde{C} \bar{K} L_{1} \ldots L_{v-1} \sum_{i=1}^{v-1} \frac{1}{L_{i}}
$$

is a function which is superadditive separately in each variable $L_{1}, \ldots, L_{v-1}$. On the other hand, its absolute value has a bound proportional to $L_{1}, \ldots, L_{v-1}$. With these 
conditions the limit

$$
\lim _{L \rightarrow \infty} \frac{g\left(L_{1}, \ldots, L_{v-1}\right)}{\prod_{i=1}^{v-1} L_{i}}=\lim _{L \rightarrow \infty} \frac{f\left(L_{1}, \ldots, L_{v-1}\right)}{\prod_{i=1}^{v-1} L_{i}}
$$

exists (see for instance [15]), which concludes the proof of the theorem.

\section{References}

1. Wegner,F.G.: J. Math. Phys. 12, 2259 (1971)

2. Messager, A., Miracle-Sole,S.: Commun. math. Phys. 40, 187 (1975)

3. Benettin, G., Gallavotti, G., Jona-Lasinio, G., Stella, A.L.: Commun. math. Phys. 30, 45 (1973)

4. Gruber, C., Hintermann, A.: Physica 83A (1975)

5. Merlini,D., Gruber,C.: J. Math. Phys. 13, 1814 (1972)

6. Gruber,C.: In: Proc. of the 2nd International Colloquium on Group Theoretical Methods in Physics, Nijmegen, Vol. 2, p. B 120-130 (1973)

7. Slawny,J.: Commun. math. Phys. 46, 75 (1976)

8. Gruber,C., Lebowitz,J.L.: Commun. math. Phys. 41, 11 (1975)

9. Brascamp, H.J.: Commun. math. Phys. 18, 82 (1970)

10. Holsztyǹski,W., Slawny,J.: Phase transitions in ferromagnetic spin systems at low temperatures. Preprint (1976)

11. Gruber,C., Greenberg,W., Merlini,D.: Physica 65, 28 (1973)

12. Gallavotti,G., Miracle-Sole,S.: Commun. math. Phys. 7, 274 (1968)

13. Lanford,O.E.: In: Mathematical methods in statistical mechanics, (ed. A. Lenard). BerlinHeidelberg-New York: Springer 1973

14. Gallavotti,G., Martin-Löf, A., Miracle-Sole, S.: In : Mathematical methods in statistical mechanics, (ed. A. Lenard). Berlin-Heidelberg-New York: Springer 1973

15. Ruelle,D.: Statistical mechanics. New York: Benjamin 1969

16. Lebowitz,J.L.: Commun. math. Phys. 28, 313 (1972)

17. Camp,W.J., Fisher,M.E.: Phys. Rev. B6, 946 (1972)

18. Lebowitz,J.L.: Coexistence of phases in Ising ferromagnets. Preprint, I.H.E.S. (1976)

Communicated by J. L. Lebowitz

Received October 17, 1976 
